Proceedings of the International Symposium on Physics of Materials (ISPMA 14), September 10-15, 2017, Prague

\title{
Molecular Dynamics Simulations of Poly(dimethylsiloxane) Elasticity
}

\author{
J. FojtíKOVÁ* And L. KalvodA \\ Czech Technical University in Prague, Faculty of Nuclear Science and Physical Engineering, \\ Břehová 7, 11519 Prague 1, Czech Republic
}

\begin{abstract}
Cross-linked polymers have unique and advantageous properties due to the infinite elastic chains. Poly(dimethylsiloxane) (PDMS) belongs into a group of non-toxic, relatively inert and highly elastic polymers (elastomers). In addition, this material is easy to fabricate and has favorable optical and mechanical properties, and it is widely used in fiber optics. Based on testing three different simulation techniques for getting closer insight into the structural background of physical properties of PDMS resin, the molecular dynamics method is chosen. The main topic of this paper is an analysis of relationship between the PDMS cross-linking level and its elasticity. The calculations are performed within the Materials Studio (MS) simulation environment (Biovia Software Inc. USA) using molecular dynamics (MD) theory implemented in MS Forcite Plus module. The obtained results are compared with the newest experimental data available for real PDMS materials.
\end{abstract}

DOI: 10.12693 /APhysPolA.134.857

PACS/topics: 31.15.xv, 61.41.+e, 81.05.Lg

\section{Introduction}

Poly(dimethylsiloxane) (PDMS) is the most used polymer from the organosilicone group. For many years, it plays an important role in food (gas permeability), cosmetic (anti-foaming agent) and medical industry (contact lenses). This material has appropriate optical properties for fiber optics applications [1]. Siloxanes generally damage the environment but the PMDS is bio-compatible, non-toxic and relatively inert [2]. In last few years there has been growing interest in mechanical properties of the PDMS because of microfluidic devices (lab-on-a-chip, microelectro-mechanical system - MEMS) [3]. PDMS is deformable (very small forces could be measured) and it easily keeps the shape of a form pattern with a great differentiation (in the $\mathrm{nm}$ ) [4]. Consequently, it is a great material for microfluidics preparation and other imprinting techniques. It is inexpensive and very easily to fabricate at a wide range of crosslinking percentage. It is in amorphous state at normal room conditions.

Our laboratory is focused on fiber and planar optic sensors where the PDMS is used as a second cladding. These sensors can measure a low concentration of gases (e.g. $\mathrm{NH}_{3}$ ). During testing measurements with sensors, we observed high sensitivity to small changes in ambient pressure. We assume that this effect could be caused by change of polymer elasticity at different crosslinking rate or due to $\mathrm{H}_{2} \mathrm{O}$ vapors adsorbed on PDMS surface.

In this paper, we attempt to verify the first hypothesis using molecular dynamics simulations. The elasticity of a polymer is a very complex problem, thus for simplicity

\footnotetext{
* corresponding author; e-mail: jaroslava.fojtikova@fjfi.cvut.cz
}

we ignore surface effects and try to map bulk modulus dependency on crosslinking rate only. All calculations are performed within Materials Studio (MS, BIOVIA Software) environment [5].

\section{Simulation method}

The classical equations of motion are the basis of molecular dynamics simulations. They are modified to deal with the effects of temperature and pressure on a system (defined statistical ensemble, NPT).

Our system consists of one large molecule of PDMS with different topology as shown in Fig. 1. This molecule (as a group of linear and crosslinker fragments) is put into a cubic cell of defined density $\left(0.965 \mathrm{~g} \mathrm{~cm}^{-3},[1]\right)$ using module MS Amorphous Cell. The crosslinker fragment is chosen in accordance with composition of commercial Sylgard 184 resin (Dow Corning). After that the fragments are joint together using special program simulating a condensation reaction of polymer controlling physical properties as volume and temperature. Inter- and intramolecular forces in the system are described by Compass II force field that is the most validated and used for polymers and composite materials. Surface effects are eliminated due to the implemented periodic boundary conditions of the cell.

Then, the cubic structure (Fig. 2) is geometrically optimized (ABNR and Quasi-Newton methods) and annealed in the temperature range $200-600 \mathrm{~K}$ in order to confirm presence of a global energetic minimum of the system.

After that, molecular dynamics of canonical ensemble $(\mathrm{N}, \mathrm{V}, \mathrm{T}=$ const. $)$ is performed on the prepared testing samples at room temperature.

Finally, NPT dynamics is used to compress the testing samples to the defined pressure and temperature states $(0.1,0.4,0.8,1.0 \mathrm{GPa}, 298 \mathrm{~K})$, under control of the Berendsen barostat and Nose thermostat methods. 


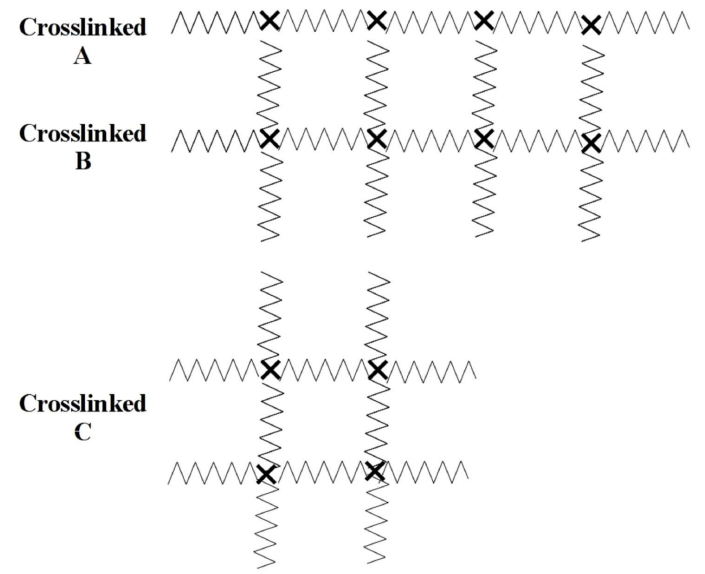

Fig. 1. Three types of polymer topology. Small crosses denote the positions of crosslinkers.

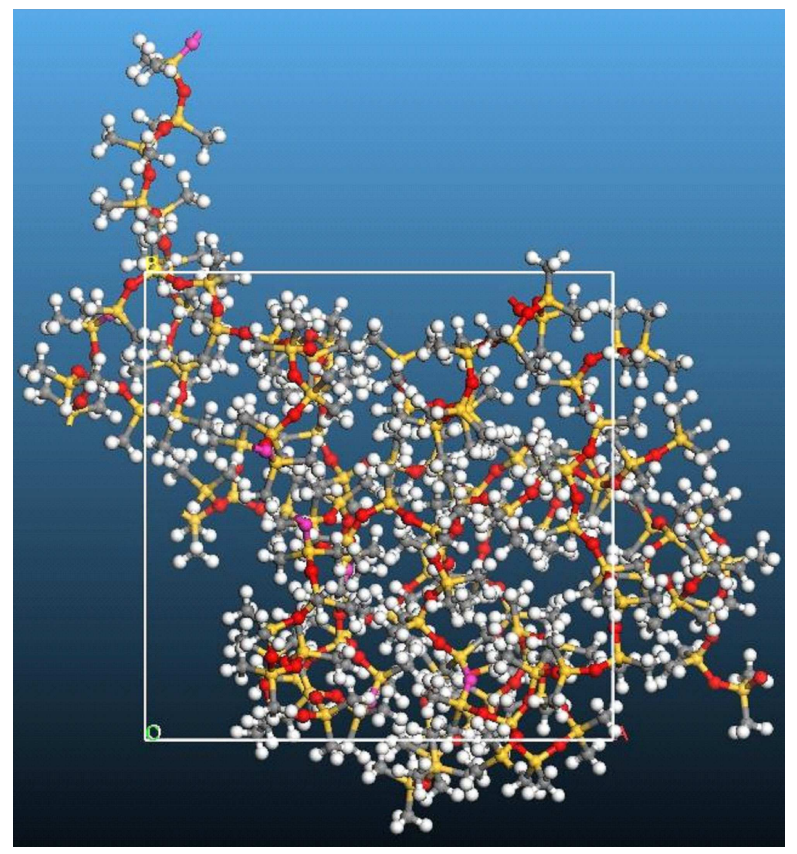

Fig. 2. The cubic cell with crosslinked PDMS topology C.

The bulk moduli of the equilibrated structures are analyzed using the constant strain method with the strain of $0.3 \%$ applied in six basic directions of cubic cell.

The whole procedure did not provide an equilibrated structure at the desired pressure level of $0.1 \mathrm{MPa}$, thus the $K$ modulus was estimated according to the formula $K=\rho_{0}(\mathrm{~d} P / \mathrm{d} \rho)_{\rho 0}$ where $\rho_{0}$ is the experimental density at the given $T$ and the fixed number of monomer units present in the chain fragments.

\section{Results and discussion}

The newest experimental values of elastic modulus of PDMS reported for the Sylgard 184 resin prepared under standard recommended conditions (curing time and tem- perature) vary from 0.1 to $1.75 \mathrm{MPa}$ with the crosslinking ratio growing from 1.96 to $9.0 w t . \%$. These results were obtained from macroscale compression tests and microscale nano-JKR experiments [2].

If we assume an isotropic structure and the Possion ratio of PDMS ca. 0.45 [2], the bulk modulus should amount ca. $6 \mathrm{MPa}$. The latter value is expected to be rising up with the crosslinking ratio growing.

The simulated values of the $K$ modulus calculated for three selected topologies are summarized in Table I. NPT dynamics parameters are as follows: $t=50 \mathrm{ps}$, $P=0.1,0.4,0.8,1.0 \mathrm{GPa}$.

TABLE I

Dependence of the elastic parameter $K$ on the crosslinking ratio with detailed parameters of NPT MD.

\begin{tabular}{c|c|c}
\hline \hline $\begin{array}{c}\text { PDMS } \\
\text { form }\end{array}$ & $\begin{array}{c}\text { Crosslinking } \\
\text { [wt.\%] }\end{array}$ & K [MPa] \\
\hline cross A & 25 & $(0.81 \pm 0.15)$ \\
cross B & 13 & $(0.75 \pm 0.10)$ \\
cross C & 11 & $(0.55 \pm 0.16)$
\end{tabular}

In spite of performing the $K$ modulus calculations on the most equilibrate structures, the obtained values are still underestimated and the system seems to be "softer" than real experimental samples.

On the other hand, the calculations confirmed the expected increase of $K$ with the rising crosslinking ratio value. The results seem to indicate that elastic properties of PDMS depend in the major extend on the crosslinking ratio and the particular network topology is of a less importance. This hypothesis will be verified on more extensive models featuring a range of different and more complicated topologies.

In the future, we plan to simulate elastic properties of PDMS using mesoscale or finite element analysis methods compatible with the known viscoelastic behavior of PDMS, the viscoelasticity might be one of the reasons why $K$ modulus values are systematically underestimate in the MD simulations.

\section{Acknowledgments}

The research was supported by the Czech Ministry of Education, Youth and Sports grant RVO14000, the Student grant system of the Czech Technical University in Prague grant No. SGS16/245/OHK4/3T/14, and the Czech Science Foundation grant 14-36566G.

\section{References}

[1] J.C. Lottersy, W. Olthuis, P.H. Veltink, P. Bergveld, J. Micromech. Microeng. 7, 145 (1997).

[2] A. Sharfeddin, A.A. Volinsky, G. Mohan, N.D. Gallant, J. Apll. Polym. Sci. 132, 42680 (2015).

[3] F. Schneider, T. Fellner, J. Wilde, U. Wallrabe, J. Micromech. Microeng. 18, 065008 (2008).

[4] G.V. Casquillas, T. Houssin, PDMS: A review, Elveflow.

[5] BIOVIA, Materials Studio. 